\title{
LIVER TRANSPLANTATION IS A PREFERABLE ALTERNATIVE TO PALLIATIVE THERAPY FOR SELECTED PATIENTS WITH ADVANCED HEPATOCELLULAR CARCINOMA
}

\author{
Aloysious D Aravinthann, ${ }^{12}$, Silvio G Bruniं, Adam C Doyle ${ }^{1}$, Hla-Hla Thein ${ }^{4}$, Nicolas Goldaracena ${ }^{1}$, \\ Assaf Issachar ${ }^{1}$, Leslie B Lilly ${ }^{1}$, Nazia Selzner ${ }^{1}$, Mamatha Bhat ${ }^{1}$, Boraiah Sreeharsha ${ }^{2}$, Markus \\ Selzner ${ }^{1}$, Anand Ghanekar ${ }^{1}$, Mark S Cattral ${ }^{1}$, Ian D McGilvray ${ }^{1}$, Paul D Greig ${ }^{1}$, Eberhard L Renner ${ }^{1}$, \\ David R Grant ${ }^{1}$, Gonzalo Sapisochin ${ }^{1}$
}

\begin{abstract}
${ }^{1}$ Multiorgan Transplant Program, Toronto General Hospital, University of Toronto, Toronto, Canada ${ }^{2}$ National Institute for Health Research (NIHR) Nottingham Digestive Diseases Biomedical Research Unit, University of Nottingham and Nottingham University Hospitals NHS Trust, Nottingham, UK 3Department of Medical Imaging, University of Toronto, Canada ${ }^{4}$ Dalla Lana School of Public Health, University of Toronto, Canada
\end{abstract}

\section{ABSTRACT}

Background: Patients with hepatocellular carcinoma (HCC) beyond the traditional criteria (advanced $\mathrm{HCC}$ ) are typically offered palliation, which is associated with a 3 -year survival rate lower than $30 \%$. This study aimed to describe the outcomes for a subset of patients with advanced HCC who satisfied the Extended Toronto Criteria (ETC) and were listed for liver transplantation (LT).

Materials \& Methods: All patients listed in the Toronto liver transplant program with HCC beyond both the Milan and University of California, San Francisco criteria were included in this study. Data were extracted from the prospectively collected electronic database. All radiological images were reviewed by two independent radiologists. The primary endpoint was patient survival.

Results: Between January 1999 and August 2014, 96 patients with advanced HCC were listed for LT, and $62(65 \%)$ of these patients received bridging therapy while on the waiting list. Bridging therapy led to a significant reduction in tumor progression $(p=0.02)$ and tumor burden $(p<0.001)$. The majority of those listed underwent $L T(n=69,72 \%)$. Both tumor progression on waiting list (HR 4.973 $[1.599-15.464], p=0.006)$ and peak AFP $\geq 400 \mathrm{ng} / \mathrm{ml}(\mathrm{HR} 4.604$ [1.66o - 12.768], $p=0.003$ ) were independently associated with waiting list dropout. Post-LT HCC recurrence occurred in $35 \%(n=24)$. Among those with HCC recurrence, survival was significantly better for those who received curative treatment $(p=0.004)$. The overall actuarial survival rates from the listing were $76 \%$ at 1 year, $56 \%$ at 3 years, and $47 \%$ at 5 years, and the corresponding rates from $L T$ were $93 \%, 71 \%$, and $66 \%$.

Conclusion: LT provides significantly better survival rates than palliation for patients with selected advanced HCC.

\section{SYNOPSIS}

In most Western centers, palliation remains the only option for patients with advanced $\mathrm{HCC}$ with a 3year survival $<30 \%$. This study reports an improved survival with liver transplantation $(71 \%, 66 \%$ at 3 , 5 years) of selected patients with advanced HCC.

\section{POSTSCRIPT}

This study was presented as an oral presentation in the International Liver Transplantation Society (ILTS) $22^{\text {nd }}$ annual international congress (2016) held in Seoul, South Korea. 


\section{INTRODUCTION}

Besides being a potentially curative treatment for early hepatocellular carcinoma (HCC) $[1,2]$, liver transplantation (LT) has the added advantage of removing the underlying cirrhosis and thereby minimizing the future risk of de novo HCC. Since the introduction of Milan criteria in 1996 [3], a postLT recurrence of $<15 \%$ and a 5 -year survival of $>70 \%$ have become the acceptable norm $[3,4]$. A number of expanded criteria have since been proposed, and shown to achieve comparable outcomes. The most widely recognized are the University of California, San Francisco (UCSF) criteria $[5,6]$, which are associated with a recurrence of $\sim 10 \%$ and a 5 -year survival of $75-80 \%$. Generally, LT is not recommended for patients with advanced $\operatorname{HCC}[1,2,7]$ due to previous undesirable experience (high recurrence and poor survival) with unrestricted access [8].

In most Western centers, palliative therapy remains the only treatment option for patients with advanced HCC. Locoregional modalities such as transarterial chemoembolization (TACE), stereotactic body radiation (SBRT) and systemic therapy (Sorafenib) are used for palliation, resulting in a 3-year survival of $<30 \%$ at best $[9,10]$.

Improvements in understanding $\mathrm{HCC}$ biology and its role in predicting outcomes has rekindled the interest in LT for advanced HCC [11]. Encouragement from the improved outcomes with LT for HCC and dissatisfaction with the poor outcomes of palliative therapy has led to reexamination of the role of $L T$ in advanced HCC.

The Extended Toronto Criteria (ETC) place no restriction on the number HCC lesions or tumor size. Patients with any number and any size of HCC lesions are eligible for LT no evidence exists for vascular invasion or extrahepatic disease, no cancer-related constitutional symptoms are observed, and a targeted biopsy of the largest lesion does not show poor differentiation. Findings show that the LT outcomes for those beyond Milan but within the ETC were inferior compared to those within Milan, but were still acceptable [12]. However, no studies have specifically focused on the outcomes of patients with advanced HCC from the time of listing as reported herein. This study aimed to investigate and report on the outcomes of patients listed for LT with advanced HCC (within ETC) who would have received palliative treatment in most Western centers. 


\section{MATERIALS AND METHODS}

\section{Study population}

The analysis included patients listed in the Toronto liver transplant program for advanced HCC, patients listed for non-advanced HCC who had progressed to advanced HCC while on the waiting list, and patients listed for non-HCC indications who had developed advanced HCC while on the waiting list. Advanced HCC was defined using pre-transplant imaging demonstrating tumors beyond both Milan and UCSF criteria, but within the ETC.

Demographic and clinical data were retrospectively extracted from the prospectively collected electronic transplant database. The study was approved by the Research Ethics Board (15-9989-CE).

\section{Assessment of change in tumor burden}

All patients underwent radiological imaging every 3 months, irrespective of bridging therapy and were independently reviewed by radiologists (S.G.B. and B.S.) blinded to the outcome. Tumor burden was assessed according to the modified Response Evaluation Criteria in Solid Tumors (mRECIST) [13]. At baseline, HCC lesions were divided into target and non-target lesions based on their longest diameter. Up to five lesions (instead of two lesions as specified by mRECIST), each measuring $>1 \mathrm{~cm}$ in at least one dimension were identified as target lesions. In the event of $>5$ lesions each measuring $>1 \mathrm{~cm}$, the largest five lesions were identified as target lesions. All other lesions were identified as non-target lesions.

Change in both target and non-target lesions were considered in assessing the overall change in tumor burden. In the event of bridging therapy, only the maximum length of the viable tumor was measured. The overall change in tumor burden was reported according to mRECIST criteria as complete response, partial response, stable disease, or progressive disease (summarized in Supplementary Table 1).

\section{Bridging therapy}

All listed patients were assessed for bridging therapy at the weekly multidisciplinary hepatoma board meetings. Patients without decompensation who were expected to have $>6$ months wait time underwent bridging therapy based on consensus opinion. In general, percutaneous ablation was recommended for lesions measuring $\leq 3 \mathrm{~cm}$; TACE was recommended for non-ablatable lesions with satisfactory liver function, and SBRT was considered for lesions not amenable to ablation and TACE. Surgical resection was not used as a bridging therapy, but patients with prior resection were included.

The utility and technology of bridging therapy modalities have evolved during the study period. In brief, these include (1) relative obsolescence of percutaneous ethanol injection for tumor ablation, (2) use of drug-eluting bead TACE over conventional TACE, (3) routine practice of superselective TACE, where possible, to maximize the impact on tumor and minimize the liver injury, and (4) the routine use of SBRT as a bridge to LT (since 2006) in patients not eligible for ablation and TACE [14].

\section{Waiting list dropout}

Patients who experienced death or tumor progression beyond the ETC while awaiting transplant were considered dropouts. Those who remained within ETC despite tumor progression were not delisted. 


\section{Endpoints}

The primary endpoint was patient survival. This was defined from the time of listing and transplantation (for those who underwent LT) to dropout or death. Survivors were censored at the time of their last clinic visit.

\section{Data analysis and statistics}

Data are shown as median (interquartile range) or number (percentage) unless otherwise stated. Statistical analyses were performed using either GraphPad prism 5 (San Diego, CA) or SPSS for Windows v20. A p-value of $<0.05$ was considered significant. For analysis purposes, peak AFP was reclassified as a binary variable of $<400 \mathrm{ng} / \mathrm{ml}$ or $\geq 400 \mathrm{ng} / \mathrm{ml}$, as previously reported [15]; overall change in tumor burden was reclassified as progressive disease or non-progressive disease (includes complete response, partial response, and stable disease). Univariate analysis with Mann-Whitney U test or 1-way ANOVA (Kruskal-Wallis test) was undertaken to identify risk factors associated with waiting list outcome (transplantation versus dropout). A multivariable logistic regression model incorporating variables with a $\mathrm{p}$-value of $<0.10$ on univariate testing was used to determine independent associations with waiting list dropout. Survival and disease recurrence estimates were calculated using univariate Cox proportional hazard models. Variables with a $p$-value of $<0.10$ on univariate testing were included in a multivariable Cox proportional hazard model. In all multivariable analyses, variables were incorporated using a backward selection process, with variables retained in the model if they maintained statistical significance. 


\section{RESULTS}

\section{Waiting list characteristics}

\subsection{Demographics}

Between January 1999 and August 2014, 96 listed patients fulfilled the inclusion criteria (Table 1). The median age at listing was 59 years (IOR $52-63)$ and the majority $(n=83,86 \%)$ were men. Chronic Hepatitis $C(n=41,43 \%)$ was the most common etiology of liver disease, followed by chronic Hepatitis $B$, alcohol-related liver disease and non-alcohol-related steatohepatitis.

\subsection{Tumor burden}

At baseline, the median total number of HCC lesions (target and non-target lesions) was 5 (IOR $3-8$ ), the median diameter of the largest lesion was $4.4 \mathrm{~cm}$ (IQR $2.6-6.3$ ), and the median total diameter of target lesions was $8.9 \mathrm{~cm}$ (IOR $6.4-12.7$ ). Immediately before to LT/dropout, the median total number of HCC lesions was 6 (IQR $2-11$ ), the median diameter of the largest lesion was $3.0 \mathrm{~cm}$ (IQR $1.2-5.8)$, and the median total diameter of target lesions was $6.3 \mathrm{~cm}$ (IQR $1.2-11.4)$. More than half $(n=55,57 \%)$ had progressive disease with or without bridging therapy.

\subsection{Bridging therapy}

Nearly two thirds of the patients $(n=62,65 \%)$ received bridging therapy. The majority $(n=49,79 \%)$ received bridging therapy by a single method. The median number of bridging therapy sessions was $2(\operatorname{IOR} 1-2)$ of any modality.

The most common method was TACE $(n=47,76 \%)$, used either alone $(n=38,61 \%)$ or in combination with other methods $(n=9,15 \%)$. Radiofrequency ablation was used for 18 patients $(29 \%)$, whereas percutaneous ethanol injection therapy and SBRT, either alone or in combination, were used for four patients (6\%).

A comparison between patients who received bridging therapy and those who did not is summarized in Table 2. The distribution of etiology of liver disease was significantly different $(p=0.02)$ between the two groups - hepatitis B was common in those who received bridging therapy, whereas hepatitis $C$ and alcohol-related liver disease were more common among those who did not. The severity of liver disease (based on Sodium-MELD score) was less $(p<0.001)$ in patients who received bridging therapy. Both peak AFP and tumor burden (total number of HCC lesions, diameter of largest target lesion and total diameter of all target lesions) at listing were similar between the two groups. As expected, bridging therapy led to a significant reduction in tumor burden. Receiving bridging therapy per se did not impact the waiting list outcome. However, tumor progression on waiting list was evident in a significantly greater proportion of patients who did not receive bridging therapy $(n=25$, $74 \%$ vs. $n=30,48 \% ; p=0.02$ ).

\section{Waiting list outcome - transplantation versus dropout}

Nearly three fourths $(n=69,72 \%)$ underwent $L T$; majority received a deceased donor graft $(n=57$, 83\%). All but one dropped out due to tumor progression beyond the ETC - extrahepatic spread $(n=10)$, macrovascular invasion $(n=9)$, tumor progression with development of cancer-related symptoms $(n=4)$, and tumor rupture $(n=3)$. 
In the univariate analysis (Table 3), the tumor burden at listing did not differ between the groups. Female sex $(p=0.03)$ and peak AFP $\geq 400 \mathrm{ng} / \mathrm{ml}(p=0.0007)$, tumor burden at $L T / d r o p o u t$, and tumor progression on the waiting list $(p=0.03)$ were associated with dropout.

Multivariate analysis was performed using sex, duration on waiting list, peak AFP $>400 \mathrm{ng} / \mathrm{ml}$, and tumor progression on waiting list as explanatory variables. Other significant variables in the univariate analysis were not included as they either measured concurrently with the outcome (tumor burden at LT/dropout), or were reflected in a statistically more powerful way using categorical variables (change in tumor burden). In the multivariate analysis, both tumor progression on waiting list (HR 4.973 [1.599-15.464], $p=0.006)$ and peak AFP $\geq 400 \mathrm{ng} / \mathrm{ml}(\mathrm{HR} 4.604$ [1.66o-12.768], $p=0.003$ ) were independently associated with dropout.

\section{Post-LT HCC recurrence}

More than a third of the patients who had transplantation $(n=24,35 \%)$ developed recurrence after a median time of 17 months (IQR $8-25)$. The cumulative risk of recurrence was $13 \%$ at 1 year, 34\% at 3 years, and $41 \%$ at 5 years. In the univariate analysis, no tested variables were associated with recurrence (Supplementary Table 2).

Lungs $(n=9)$ were the most common site of HCC recurrence followed by bone $(n=8)$, liver $(n=5)$, and lymph nodes $(n=5)$, whereas adrenal glands and abdominal wall were infrequent sites of recurrence. All patients with recurrence were considered for curative treatment (surgery or ablation). Seven (29\%) patients underwent curative treatment (surgery, ablation), while 8 (33\%) underwent non-curative treatment (radiotherapy, palliative surgery, Sorafenib treatment), and the rest $(n=9$, $38 \%$ ) received best supportive care.

The median survival period from the time of LT (6o months [IOR $37-70$ ] vs. 27 months [IOR 14-32], $p=0.004$ ) and the median survival from the time of recurrence ( 32 months [IOR 25-36] vs. 7 months [IOR $5-13], p=0.004$ ) were significantly better for those who received curative treatment compared to others (figure 2).

\section{Survival from the time of listing and LT}

The median follow-up from the time of listing (all 96 patients) was 34 months (IOR $13-68$ ). The actuarial patient survival rate was $76 \%$ at 1 year, $56 \%$ at 3 years, and $47 \%$ at 5 years from listing.

The median follow-up from the time of LT (69 patients) was 39 months (IOR $24-80$ ). The actuarial survival rate was $93 \%$ at 1 year, $71 \%$ at 3 years, and $66 \%$ at 5 years from LT. Survival from the time of LT was not associated with any of the tested variables (Supplementary Table 3).

The number of living donor LT has remained relatively unchanged despite the gradual increase in the number of deceased donor LT for advanced HCC. Overall survival from the time of listing ( 48 vs. 50 months; $p=0.86$ ) and from the time of transplantation (39 vs. 45 months; $p=0.93$ ) did not differ between deceased donor and living donor LT. 


\section{DISCUSSION}

This study evaluates the outcomes of patients listed for LT with advanced HCC who would have received palliative treatment at most Western centers. One fourth of the listed patients dropped out; peak AFP $\geq 400 \mathrm{ng} / \mathrm{ml}$ and tumor progression on waiting list independently predicted dropout. The $5^{-}$ year post-LT survival was $66 \%$.

The patient survival of this series is lower than those within Milan criteria [12], but much better than the reported outcomes with palliative treatment, whether taken from the time of transplantation ( $71 \%$ at 3 years, $66 \%$ at 5 years) or from the time of listing ( $56 \%$ at 3 years, $47 \%$ at 5 years). For selected patients with non-transplantable HCC (i.e. beyond the Milan criteria), TACE barely accomplished a 3year survival rate of $30 \%$ even in experienced centers $[9,10]$. The use of TACE with External Beam Radiotherapy [16] or Sorafenib [17, 18] also failed to demonstrate a satisfactory patient survival. Suboptimal survival (3-year survival of $\sim 20 \%$ ) has also been reported with Yttrium-9o selective internal radiation therapy in patients with intermediate-stage HCC deemed unsuitable for LT [19-21]. Thus, in the absence of comparable treatment options, it is not unreasonable to consider transplantation as a preferred alternative. On the other hand, it is necessary to be aware that the costs associated with LT are significantly higher than palliative therapies [22]. The estimated average total health care cost ( 2013 US\$) of LT for HCC is approximately $\$ 211,286$ (95\% Cl $\$ 193,919$ $\$ 228,654)$. The cost of palliative TACE is approximately $\$ 39,294(95 \% \mathrm{Cl} \$ 20,455-\$ 58,133)$ and systemic therapy (Sorafenib) is approximately $\$ 45,802(95 \% \mathrm{Cl} \$ 32,143-\$ 59,461)$ (personal communication - Hla-Hla Thein). However, despite the increased costs, the quality-adjusted life year is likely better with LT than with palliative treatments.

As clinical practice moves towards personalized medicine, it becomes more crucial that all treatment options are presented to the patients and their families. Transplantation seems a better choice for selected patients with advanced HCC, but it is limited by the scarce resource of donated organs. Deciding when it is acceptable to allocate deceased donor grafts to patients with advanced HCC will depend on the regional waiting list and organ availability. Expansion of HCC transplant criteria can be justified only if it upholds fair organ allocation. Introduction of direct anti-viral agents against hepatitic $\mathrm{C}$ is expected to increase the availability of grafts [23]; thus expansion of HCC criteria may become feasible [24]. However, this opportunity may never materialize due to the impending steatohepatitis epidemic [25]. Another alternative would be living donor LT. This does not affect the candidacy of others, and the outcomes are comparable to deceased donor LT [26, 27]; therefore, would be a preferred option for selected patients with advanced HCC. The equivalence of HCC recurrence and patient survival between deceased donor and living donor LT of the present analysis further supports this notion. However, living donor LT must be undertaken in centers with extensive experience due to safety concerns of donors [28].

The waiting list dropout was almost 30\%. Higher dropout rates have also been reported previously in patients with HCC beyond the traditional criteria [29,30]. Both AFP and tumor progression (tumor biological factors), but not tumor burden (size and numbers) were independently associated with dropout. The predictive role of tumor biology over tumor burden also has been shown in previous studies. In a recent study from the USCF group, AFP $\geq 500 \mathrm{ng} / \mathrm{ml}$ and rapid tumor progression predicted dropout even in patients with very early stage HCC (single tumor $<2 \mathrm{~cm}$ ) [31]. In another study, which included patients with HCC within and beyond Milan, the response to therapy was the 
only predictor of dropout, irrespective of tumor staging [32]. Likewise, a high AFP levels, but not total tumor volume was associated with waiting list dropout [33].

Post-transplant HCC recurrence was also found to be higher than in patients within traditional criteria. However, the 5 -year post-LT survival was $66 \%$. It is likely that the aggressive treatment of recurrence played a role in achieving such acceptable survival rates. The benefits of such aggressive treatment has also been demonstrated previously [34, 35]. The high recurrence rate may raise concerns over offering LT to this group of patients. However, it is important to recognize that patient survival, not recurrence, is the most clinically relevant and decisive outcome measure of transplantation [36].

Against expectations, the duration on waiting list did not impact post-LT survival. This contradicts the recent suggestion of implementing a period of observation to identify unfavorable tumor behavior before listing/transplantation [37-39]. One likely explanation for this discrepancy is the unaccounted period between the diagnosis of HCC (by the Hepatologist/Gastroenterologist/Family Physician) and listing, resulting in a bias towards the null. A substantial number of patients progress beyond the ETC during this period and are turned-down before they are formally assessed for LT.

This study has strengths and limitations. The use of radiological parameters rather than explant parameters in the analysis made the findings reflect prospective decision-making in day-to-day practice. On the other hand, being a single center, retrospective study with relatively limited number of patients should be considered a limitation. Some comparisons may have been affected by the relatively small cohort. In addition, during the 15 -year study period, there were significant advancements in bridging therapy and a shift toward a more aggressive management of HCC for patients on the waiting list, which had the potential for an impact on the results. Independent validation of this study results is therefore needed.

Efforts to expand the traditional HCC allocation criteria is already underway [24, 40]. One such example would be the introduction of 'Up-to-seven' criteria by Mazzaferro (who initially proposed the Milan criteria) and colleagues [41]. Similarly, the emphasis on tumor biology (such as tumor differentiation, tumor response to bridging/downstaging therapies and AFP) rather than tumor burden in selecting patients for LT is gaining momentum [11, 24, 40, 42]. Therefore, it is not unreasonable to speculate that LT will emerge as an acceptable treatment choice for selected patients with advanced HCC in the near future. Certain transplant programs in Turkey and India seem to follow an unrestricted tumor burden policy, similar to that of the ETC [43]. It is crucial that these programs publish their long-term outcome results, which may further encourage other transplant programs to expend their allocation policy. In addition, the future of LT for advanced HCC relies on the availability of donor organs and living donor LT likely will play a crucial role in this. Where available, the option of living donor LT should be discussed and offered to all patients with advanced HCC.

In conclusion, LT offers a 5 -year survival rate of $66 \%$ for selected patients with advanced $\mathrm{HCC}$ and is a preferable alternative to palliation. Living donor LT may be the best option for this cohort as it will not negatively impact others on the waiting list. 


\section{REFERENCES}

1. Bruix, J., M. Sherman, and D. American Association for the Study of Liver, Management of hepatocellular carcinoma: an update. Hepatology, 2011. 53(3): p. 1020-2.

2. European Association For The Study Of The, L., R. European Organisation For, and C. Treatment Of, EASL-EORTC clinical practice guidelines: management of hepatocellular carcinoma. J Hepatol, 2012. 56(4): p. 908-43.

3. Mazzaferro, V., et al., Liver transplantation for the treatment of small hepatocellular carcinomas in patients with cirrhosis. N Engl J Med, 1996. 334(11): p. 693-9.

4. Mazzaferro, V., et al., Milan criteria in liver transplantation for hepatocellular carcinoma: an evidence-based analysis of 15 years of experience. Liver Transpl, 2011. 17 Suppl 2: p. S44-57.

5. Yao, F.Y., et al., Liver transplantation for hepatocellular carcinoma: expansion of the tumor size limits does not adversely impact survival. Hepatology, 2001. 33(6): p. 1394-403.

6. Yao, F.Y., et al., Liver transplantation for hepatocellular carcinoma: validation of the UCSFexpanded criteria based on preoperative imaging. Am J Transplant, 2007. 7(11): p. 2587-96.

7. Bruix, J., M. Reig, and M. Sherman, Evidence-Based Diagnosis, Staging, and Treatment of Patients With Hepatocellular Carcinoma. Gastroenterology, 2016. 150(4): p. 835-53.

8. Iwatsuki, S., et al., Role of liver transplantation in cancer therapy. Ann Surg, 1985. 202(4): p. 401-7.

9. Llovet, J.M., et al., Arterial embolisation or chemoembolisation versus symptomatic treatment in patients with unresectable hepatocellular carcinoma: a randomised controlled trial. Lancet, 2002. 359(9319): p. 1734-9.

10. Lo, C.M., et al., Randomized controlled trial of transarterial lipiodol chemoembolization for unresectable hepatocellular carcinoma. Hepatology, 2002. 35(5): p. 1164-71.

11. Cillo, U., et al., Prediction of hepatocellular carcinoma biological behavior in patient selection for liver transplantation. World J Gastroenterol, 2016. 22(1): p. 232-52.

12. Sapisochin, G., et al., The extended toronto criteria for liver transplantation in patients with hepatocellular carcinoma: A prospective validation study. Hepatology, 2016.

13. Lencioni, R. and J.M. Llovet, Modified RECIST (mRECIST) assessment for hepatocellular carcinoma. Semin Liver Dis, 2010. 30(1): p. 52-60.

14. Sandroussi, C., et al., Radiotherapy as a bridge to liver transplantation for hepatocellular carcinoma. Transpl Int, 2010. 23(3): p. 299-306.

15. Toso, C., et al., Total tumor volume and alpha-fetoprotein for selection of transplant candidates with hepatocellular carcinoma: A prospective validation. Hepatology, 2015. 62(1): p. 158-65.

16. Yang, P., et al., The Degree of Lipiodol Accumulation Can Be an Indicator of Successful Treatment for Unresectable Hepatocellular Carcinoma (HCC) Patients - in the Case of Transcatheter Arterial Chemoembolization (TACE) and External Beam Radiotherapy (EBRT). J Cancer, 2016. 7(11): p. 1413-20.

17. Zhao, Y., et al., Early sorafenib-related adverse events predict therapy response of TACE plus sorafenib: A multicenter clinical study of 606 HCC patients. Int J Cancer, 2016. 139(4): p. 92837.

18. Lencioni, R., et al., Sorafenib or placebo plus TACE with doxorubicin-eluting beads for intermediate stage HCC: The SPACE trial. J Hepatol, 2016. 64(5): p. 1090-8.

19. Lance, C., et al., Comparative analysis of the safety and efficacy of transcatheter arterial chemoembolization and yttrium-9o radioembolization in patients with unresectable hepatocellular carcinoma. J Vasc Interv Radiol, 2011. 22(12): p. 1697-705. 
20. Moreno-Luna, L.E., et al., Efficacy and safety of transarterial radioembolization versus chemoembolization in patients with hepatocellular carcinoma. Cardiovasc Intervent Radiol, 2013. 36(3): p. 714-23.

21. El Fouly, A., et al., In intermediate stage hepatocellular carcinoma: radioembolization with yttrium 90 or chemoembolization? Liver Int, 2015. 35(2): p. 627-35.

22. Thein, H.H., et al., Health care costs associated with hepatocellular carcinoma: a populationbased study. Hepatology, 2013. 58(4): p. 1375-84.

23. Belli, L.S., et al., Delisting of liver transplant candidates with chronic hepatitis $C$ after viral eradication: A European study. J Hepatol, 2016. 65(3): p. 524-31.

24. Mazzaferro, V., Squaring the circle of selection and allocation in liver transplantation for HCC: An adaptive approach. Hepatology, 2016. 63(5): p. 1707-17.

25. Loomba, R. and A.J. Sanyal, The global NAFLD epidemic. Nat Rev Gastroenterol Hepatol, 2013. 10(11): p. 686-90.

26. Olthoff, K.M., et al., Defining long-term outcomes with living donor liver transplantation in North America. Ann Surg, 2015. 262(3): p. 465-75; discussion 473-5.

27. Selzner, M., et al., Live donor liver transplantation in high MELD score recipients. Ann Surg, 2010. 251(1): p. 153-7.

28. Rossler, F., et al., Defining Benchmarks for Major Liver Surgery: A multicenter Analysis of 5202 Living Liver Donors. Ann Surg, 2016. 264(3): p. 492-500.

29. Bittermann, T., et al., Waitlist priority for hepatocellular carcinoma beyond milan criteria: a potentially appropriate decision without a structured approach. Am J Transplant, 2014. 14(1): p. $79-87$.

30. Schmitt, T.M., et al., Liver transplantation for $T_{3}$ lesions has higher waiting list mortality but similar survival compared to $T_{1}$ and $T_{2}$ lesions. Ann Hepatol, 2010. 9(4): p. 390-6.

31. Mehta, N., et al., Intention to treat outcome of $T_{1}$ hepatocellular carcinoma with the "wait and not ablate" approach until meeting T2 criteria for liver transplant listing. Liver Transpl, 2016. 22(2): p. 178-87.

32. Vitale, A., et al., Response to therapy as a criterion for awarding priority to patients with hepatocellular carcinoma awaiting liver transplantation. Ann Surg Oncol, 2010. 17(9): p. 2290302.

33. Merani, S., et al., The impact of waiting list alpha-fetoprotein changes on the outcome of liver transplant for hepatocellular carcinoma. J Hepatol, 2011. 55(4): p. 814-9.

34. Sapisochin, G., et al., Benefit of Treating Hepatocellular Carcinoma Recurrence after Liver Transplantation and Analysis of Prognostic Factors for Survival in a Large Euro-American Series. Ann Surg Oncol, 2015. 22(7): p. 2286-94.

35. Bodzin, A.S., et al., Predicting Mortality in Patients Developing Recurrent Hepatocellular Carcinoma After Liver Transplantation: Impact of Treatment Modality and Recurrence Characteristics. Ann Surg, 2016.

36. Yao, F.Y., Liver transplantation for hepatocellular carcinoma: beyond the Milan criteria. Am J Transplant, 2008. 8(10): p. 1982-9.

37. Toso, C., et al., The place of downstaging for hepatocellular carcinoma. J Hepatol, 2010. 52(6): p. 930-6.

38. Roberts, J.P., et al., Hepatocellular carcinoma: Ablate and wait versus rapid transplantation. Liver Transpl, 2010. 16(8): p. 925-9. 
39. Pomfret, E.A., et al., Report of a national conference on liver allocation in patients with hepatocellular carcinoma in the United States. Liver Transpl, 2010. 16(3): p. 262-78.

40. Mulligan, D.C., The ongoing quest to find the appropriate patients to transplant with hepatocellular carcinoma: Milan to san Francisco to Toronto and beyond. Hepatology, 2016.

41. Mazzaferro, V., et al., Predicting survival after liver transplantation in patients with hepatocellular carcinoma beyond the Milan criteria: a retrospective, exploratory analysis. Lancet Oncol, 2009. 10(1): p. 35-43.

42. Lee, H.W. and K.S. Suh, Liver transplantation for advanced hepatocellular carcinoma. Clin Mol Hepatol, 2016. 22(3): p. 309-318.

43. Moray, G., et al., Expanded criteria for hepatocellular carcinoma and liver transplantation. Transplant Proc, 2007. 39(4): p. 1171-4. 


\section{TABLES}

Table 1: Characteristics of all patients $(n=96)$ listed for liver transplantation with advanced hepatocellular carcinoma, but within the Extended Toronto Criteria.

\begin{tabular}{|c|c|c|}
\hline & & $\begin{array}{c}\text { Median or Number } \\
(\text { IQR or } \%)\end{array}$ \\
\hline \multicolumn{2}{|l|}{ Age at listing (years) } & $59(52-63)$ \\
\hline \multicolumn{2}{|l|}{ Male sex } & $83(86 \%)$ \\
\hline \multirow[t]{5}{*}{ Etiology of liver disease } & $\mathrm{HCV}$ & $41(43 \%)$ \\
\hline & HBV & $24(25 \%)$ \\
\hline & ALD & $18(19 \%)$ \\
\hline & $\mathrm{NASH}$ & $6(6 \%)$ \\
\hline & Other & $7(7 \%)$ \\
\hline \multicolumn{2}{|l|}{ Duration on waiting list (months) } & $6(3-9)$ \\
\hline \multicolumn{2}{|l|}{ Sodium-MELD at listing } & $12(9-16)$ \\
\hline \multicolumn{2}{|l|}{ Peak AFP on waiting list (ng/ml) } & $87(15-806)$ \\
\hline \multicolumn{2}{|l|}{ Peak AFP $\geq 400 \mathrm{ng} / \mathrm{ml}$} & $29(30 \%)$ \\
\hline \multirow[t]{3}{*}{ Tumor burden at listing } & Total number of HCC lesions & $5(3-8)$ \\
\hline & Diameter of the largest target lesion $(\mathrm{cm})$ & $4.4(2.6-6.3)$ \\
\hline & Total diameter of target lesions $(\mathrm{cm})$ & $8.9(6.4-12.7)$ \\
\hline \multirow[t]{3}{*}{ Tumor burden at LT or dropout } & Total number of HCC lesions & $6(2-11)$ \\
\hline & Diameter of the largest target lesion $(\mathrm{cm})$ & $3.0(1.2-5.8)$ \\
\hline & Total diameter of target lesions $(\mathrm{cm})$ & $6.3(1.2-11.4)$ \\
\hline \multicolumn{2}{|c|}{ Number of patients received bridging therapy } & $62(65 \%)$ \\
\hline \multirow[t]{4}{*}{ Modality of bridging therapy used } & TACE & $47\left(76 \%{ }^{*}\right)$ \\
\hline & RFA & $18(29 \% *)$ \\
\hline & PEI & $4\left(6 \%{ }^{*}\right)$ \\
\hline & SBRT & $4\left(6 \% \%^{*}\right)$ \\
\hline \multirow[t]{4}{*}{ Change in tumor burden } & Complete response & $7(7 \%)$ \\
\hline & Partial response & $23(24 \%)$ \\
\hline & Stable disease & $11(11 \%)$ \\
\hline & Progressive disease & $55(57 \%)$ \\
\hline \multirow{2}{*}{$\begin{array}{l}\text { Change in tumor burden } \\
\text { (reclassified) }\end{array}$} & Non-progressive disease & $41(43 \%)$ \\
\hline & Progressive disease & $55(57 \%)$ \\
\hline \multirow[t]{2}{*}{ Waiting list outcome } & Dropout & $27(28 \%)$ \\
\hline & Transplantation & $69(72 \%)$ \\
\hline \multirow[t]{2}{*}{ Donor type } & DDLT & $57\left(83 \%^{+}\right)$ \\
\hline & LDLT & $12\left(17^{\%} \%^{\dagger}\right)$ \\
\hline
\end{tabular}

AFP, alpha fetoprotein; ALD, alcohol-related liver disease; DDLT, deceased donor liver transplantation; HBV, chronic hepatitis B viral infection; HCC, hepatocellular carcinoma; $\mathrm{HCV}$, chronic hepatitis $\mathrm{C}$ viral infection; LDLT, living donor liver transplantation; LT, liver transplantation; MELD, Model for End-stage Liver Disease score; NASH, non-alcohol-related steatohepatitis; PEI, percutaneous ethanol injection; RFA, radiofrequency ablation; SBRT, stereotactic body radiation therapy; TACE, transarterial chemoembolization; UCSF, University of California San Francisco.

*Expressed as percentage of those who received bridging therapy $(n=62)$.

tExpressed as percentage of those who underwent liver transplantation $(n=69)$. 
Table 2: Comparison between patients who received bridging therapy $(n=62)$ and patients who did not receive bridging therapy $(n=34)$.

\begin{tabular}{|c|c|c|c|c|}
\hline & & \\
\hline & & Bridging therapy $(n=62)$ & No bridging therapy $(n=34)$ & \multirow[b]{2}{*}{ p-value } \\
\hline & & $\begin{array}{l}\text { median or number } \\
\text { (IOR or \%) }\end{array}$ & $\begin{array}{l}\text { median or number } \\
\quad(\text { IQR or } \%)\end{array}$ & \\
\hline \multicolumn{2}{|l|}{ Age at listing (years) } & $58(51-62)$ & $61(56-64)$ & 0.06 \\
\hline \multicolumn{2}{|l|}{ Male sex } & $54(87 \%)$ & $29(85 \%)$ & 0.80 \\
\hline \multirow[t]{5}{*}{ Etiology of liver disease } & $\mathrm{HCV}$ & $24(39 \%)$ & $17(50 \%)$ & \multirow{5}{*}{0.02} \\
\hline & HBV & $20(32 \%)$ & $4(12 \%)$ & \\
\hline & ALD & $8(13 \%)$ & $10(29 \%)$ & \\
\hline & NASH & $3(5 \%)$ & $3(9 \%)$ & \\
\hline & Other & $7(11 \%)$ & $\mathrm{o}(0 \%)$ & \\
\hline \multicolumn{2}{|l|}{ Duration on waiting list (months) } & $6(3-9)$ & $5(3-8)$ & 0.55 \\
\hline \multicolumn{2}{|l|}{ Sodium-MELD at listing } & $11(9-13)$ & $16(12-20)$ & $<0.001$ \\
\hline \multicolumn{2}{|l|}{ Peak AFP $\geq 400 \mathrm{ng} / \mathrm{ml}$} & $21(34 \%)$ & $8(24 \%)$ & 0.29 \\
\hline \multirow[t]{3}{*}{ Tumor burden at listing } & Total number of HCC lesions & $4(2-9)$ & $6(3-7)$ & 0.41 \\
\hline & Diameter of the largest target lesion $(\mathrm{cm})$ & $5.2(2.6-7.3)$ & $3.5(2.6-5.2)$ & 0.14 \\
\hline & Total diameter of target lesions $(\mathrm{cm})$ & $9.0(6.2-14.2)$ & $8.0(6.4-11.4)$ & 0.57 \\
\hline \multirow[t]{3}{*}{ Tumor burden at LT or dropout } & Total number of HCC lesion & $4(2-11)$ & $8(6-11)$ & 0.002 \\
\hline & Diameter of the largest target lesion $(\mathrm{cm})$ & $1.8(0.0-4.1)$ & $4.6(3.2-7.1)$ & $<0.001$ \\
\hline & Total diameter of target lesions $(\mathrm{cm})$ & $2.1(0.0-5.6)$ & $11.4(8.4-14.8)$ & $<0.001$ \\
\hline \multicolumn{2}{|c|}{ Progressive disease on waiting list (according to mRECIST) } & $30(48 \%)$ & $25(74 \%)$ & 0.02 \\
\hline \multirow{2}{*}{\multicolumn{2}{|c|}{$\begin{array}{ll}\text { Waiting list outcome } & \text { Dropout } \\
\text { LT }\end{array}$}} & $14(23 \%)$ & $13(38 \%)$ & \multirow{2}{*}{0.10} \\
\hline & & $48(77 \%)$ & $21(62 \%)$ & \\
\hline
\end{tabular}

Abbreviations: AFP, alpha fetoprotein; ALD, alcohol-related liver disease; HBV, chronic hepatitis B viral infection; HCC, hepatocellular carcinoma; HCV, chronic hepatitis C viral infection; LT, liver transplantation; MELD, Model for End-stage Liver Disease score; NASH, non-alcohol-related steatohepatitis. A p-value <0.05 is indicated in bold. 
Table 3: Comparison between patients who underwent liver transplantation $(n=69)$ and who dropped out $(n=27)$.

\begin{tabular}{|c|c|c|c|c|c|c|}
\hline & & \multirow{2}{*}{$\begin{array}{l}\text { Dropouts }(n=27) \\
\text { median or number } \\
(\text { IQR or } \%)\end{array}$} & \multirow{2}{*}{$\begin{array}{c}\text { Transplanted }(n=69) \\
\text { median or number } \\
\text { (IQR or \%) }\end{array}$} & \multirow[b]{2}{*}{ p-value } & \multicolumn{2}{|c|}{ Multivariate analysis } \\
\hline & & & & & hazard ratio $(95 \% \mathrm{Cl})$ & p-value \\
\hline \multicolumn{2}{|l|}{ Age at listing (years) } & $60(50-65)$ & $59(52-62)$ & 0.57 & & \\
\hline \multicolumn{2}{|l|}{ Male sex } & $20(74 \%)$ & $63(91 \%)$ & 0.03 & - & 0.39 \\
\hline \multirow[t]{5}{*}{ Etiology of liver disease } & $\mathrm{HCV}$ & $13(48 \%)$ & $28(40 \%)$ & & & \\
\hline & HBV & $7(26 \%)$ & $17(25 \%)$ & & & \\
\hline & ALD & $4(15 \%)$ & $14(20 \%)$ & 0.86 & & \\
\hline & $\mathrm{NASH}$ & $2(7 \%)$ & $4(6 \%)$ & & & \\
\hline & Other & $1(4 \%)$ & $6(9 \%)$ & & & \\
\hline \multicolumn{2}{|l|}{ Duration on waiting list (months) } & $7(4-11)$ & $5(3-8)$ & 0.08 & - & 0.75 \\
\hline \multicolumn{2}{|l|}{ Sodium-MELD at listing } & $13(9-17)$ & $11(9-16)$ & 0.57 & & \\
\hline \multicolumn{2}{|l|}{ Peak AFP $\geq 400 \mathrm{ng} / \mathrm{ml}$} & $15(56 \%)$ & $14(20 \%)$ & 0.0007 & $4.604(1.660-12.768)$ & 0.003 \\
\hline \multirow[t]{3}{*}{ Tumor burden at listing } & Total number of HCC lesions & $7(3-13)$ & $5(3-7)$ & 0.27 & & \\
\hline & Diameter of the largest target lesion $(\mathrm{cm})$ & $4.5(2.9-5.9)$ & $4.4(2.6-6.4)$ & 1.0 & & \\
\hline & Total diameter of target lesions $(\mathrm{cm})$ & $8.9(5.8-14.9)$ & $8.9(6.4-11.7)$ & 0.83 & & \\
\hline \multirow[t]{3}{*}{ Tumor burden at LT or dropout } & Total number of HCC lesions & $10(6-29)$ & $4(2-9)$ & 0.003 & & \\
\hline & Diameter of the largest target lesion $(\mathrm{cm})$ & $4.9(1.9-6.6)$ & $2.6(1.0-4.2)$ & 0.047 & & \\
\hline & Total diameter of target lesions $(\mathrm{cm})$ & $10.1(3.7-13.7)$ & $3.3(1-9.9)$ & 0.04 & & \\
\hline \multicolumn{2}{|c|}{ Progressive disease on waiting list (according to mRECIST) } & $22(81 \%)$ & $33(48 \%)$ & 0.03 & $4.973(1.599-15.464)$ & 0.006 \\
\hline
\end{tabular}

Abbreviations: AFP, alpha fetoprotein; ALD, alcohol-related liver disease; HBV, chronic hepatitis B viral infection; HCC, hepatocellular carcinoma; HCV, chronic hepatitis C viral infection; LT, liver transplantation; MELD, Model for End-stage Liver Disease score; NASH, non-alcohol-related steatohepatitis. A p-value $<0.05$ is indicated in bold. 
Supplementary Table 1: Overall change in tumor burden according to the modified Response Evaluation Criteria in Solid Tumors (mRECIST) ${ }^{+}$

\begin{tabular}{llll}
\hline Overall tumor response & Target lesion response & Non-target lesion response \\
\hline Complete response & Complete response & AND & Complete response \\
\hline Partial response & Complete response or partial response & AND & Incomplete response / stable disease \\
\hline Stable disease & Stable disease & AND & Complete response or incomplete response / stable disease \\
\hline Progressive disease & Progressive disease & OR & Progressive disease \\
\hline
\end{tabular}

${ }^{\dagger}$ Up to five lesions were identified as target lesions instead of two as with mRECIST.

Target lesion response: complete response: disappearance of any intratumoral arterial enhancement in all target lesions; partial response - at least a $30 \%$ reduction in the sum of diameter

of viable target lesions; progressive disease - at least a $20 \%$ increment in the sum of diameter of viable target lesions; stable disease - a change in the sum of diameter of viable target lesions which cannot be categorized as partial response or progressive disease.

Non-target lesion response: complete response - disappearance of intratumoral arterial enhancement in all non-target lesions; incomplete response / stable disease - persistence of

intratumoral arterial enhancement in one or more non-target lesions; progressive disease - appearance of new lesion/s or unequivocal increase in size of existing non-target lesion/s. 
Supplementary Table 2: Post-transplantation HCC recurrence $(n=69)$.

\begin{tabular}{llcc} 
& & \multicolumn{2}{c}{ Univariate analysis } \\
\cline { 3 - 4 } & & $0.984(0.936-1.033)$ & p-value \\
\cline { 3 - 4 } & & $0.629(0.187-2.110)$ & 0.45 \\
\hline Age at listing (years) & 1.0 & - \\
\hline Male sex & hCV & 0.26 \\
\hline Etiology of liver disease & HBV & $0.550(0.196-1.547)$ & - \\
& ALD & $0.629(0.205-1.931)$ & 0.42 \\
& NASH & - & 0.48 \\
\hline Duration on waiting list (months) & $0.581(0.131-2.582)$ & 0.74 \\
\hline Sodium-MELD at listing & & $1.013(0.939-1.092)$ & 0.13 \\
\hline Peak AFP $\geq 400$ ng/ml & & $0.923(0.834-1.022)$ & 0.53 \\
\hline Tumor burden at listing & Total number of HCC lesions & $1.344(0.531-3.397)$ & 0.68 \\
& Diameter of the largest target lesion & $1.009(0.969-1.050)$ & 0.37 \\
\hline Tumor burden at LT & Total diameter of target lesions & $1.056(0.938-1.189)$ & 0.31 \\
\hline & Total number of HCC lesions & $1.036(0.967-1.110)$ & 0.37 \\
\hline Progressive disease on waiting list (according to mRECIST) & $1.008(0.990-1.027)$ & 0.61 \\
\hline Donor type - living donor liver transplantation & $1.036(0.904-1.188)$ & 0.63 \\
\hline
\end{tabular}

Abbreviations: AFP, alpha fetoprotein; ALD, alcohol-related liver disease; HBV, chronic hepatitis B viral infection; HCC, hepatocellular carcinoma; HCV, chronic hepatitis C viral infection; LT, liver transplantation; MELD, Model for End-stage Liver Disease score; NASH, non-alcohol-related steatohepatitis. 
Supplementary Table 3: Patient survival from the time of transplantation $(n=69)$.

\begin{tabular}{lccc} 
& & \multicolumn{2}{c}{ Univariate analysis } \\
\cline { 3 - 4 } & & hazard ratio (95\% Cl) & p-value \\
\hline Age at listing (years) & & $0.981(0.936-1.028)$ & 0.42 \\
\hline Male sex & HCV & $0.630(0.185-2.142)$ & - \\
\hline Etiology of liver disease & 1.0 & 0.43 \\
& HBV & $0.654(0.227-1.882)$ & 0.25 \\
& ALD & $0.478(0.135-1.696)$ & 0.90 \\
& NASH & $0.871(0.112-6.757)$ & 0.55 \\
\hline Duration on waiting list (months) & $0.626(0.135-2.897)$ & 0.25 \\
\hline Sodium-MELD at listing & & $1.040(0.972-1.112)$ & 0.33 \\
\hline Peak AFP $\geq 400$ ng/ml & & $0.951(0.859-1.052)$ & 0.81 \\
\hline Tumor burden at listing & Total number of HCC lesions & $1.132(0.420-3.053)$ & 1.0 \\
& Diameter of the largest target lesion & $1.000(0.952-1.051)$ & 0.97 \\
& Total diameter of target lesions & $1.002(0.884-1.135)$ & 0.92 \\
\hline Tumor burden at LT & Total number of HCC lesions & $0.996(0.921-1.076)$ & 0.89 \\
& Diameter of the largest target lesion & $0.998(0.971-1.026)$ & 0.51 \\
\hline Progressive disease on waiting list (according to mRECIST) & $1.044(0.918-1.188)$ & 0.75 \\
\hline Donor type - living donor liver transplantation & $1.009(0.956-1.065)$ & 0.37 \\
\hline
\end{tabular}

Abbreviations: AFP, alpha fetoprotein; ALD, alcohol-related liver disease; HBV, chronic hepatitis B viral infection; HCC, hepatocellular carcinoma; HCV, chronic hepatitis C viral infection; LT, liver transplantation; MELD, Model for End-stage Liver Disease score; NASH, non-alcohol-related steatohepatitis. 


\section{FIGURE LEGENDS}

Figure 1

Flow chart representation of the study cohort $(n=96)$.

\section{Figure 2}

Kaplan-Meier survival estimates of patients who developed hepatocellular carcinoma recurrence following transplantation from the time of liver transplantation and from the time of diagnosis of recurrence. The solid line represents patients who underwent curative treatment and the interrupted line represents patients who underwent non-curative treatment. 